\title{
Review Article \\ Efficacy and Safety of L-Carnitine Treatment for Chronic Heart Failure: A Meta-Analysis of Randomized Controlled Trials
}

\author{
Xiaolong Song, ${ }^{1,2}$ Huiyan Qu, ${ }^{1}$ Zongguo Yang, ${ }^{3}$ Jingfeng Rong, ${ }^{1}$ Wan Cai, ${ }^{1}$ and Hua Zhou ${ }^{1}$ \\ ${ }^{1}$ Department of Cardiology, Shuguang Hospital, Shanghai University of Traditional Chinese Medicine, Shanghai 201203, China \\ ${ }^{2}$ Department of Cardiology, Yancheng Traditional Chinese Medicine Hospital Affiliated to Nanjing University of Chinese Medicine, \\ Yancheng 224001, China \\ ${ }^{3}$ Department of Traditional Chinese Medicine, Shanghai Public Health Clinical Center, Fudan University, Shanghai 201508, China
}

Correspondence should be addressed to Hua Zhou; zhouhuahm@163.com

Received 12 January 2017; Revised 19 March 2017; Accepted 22 March 2017; Published 13 April 2017

Academic Editor: Hai-Feng Pan

Copyright (c) 2017 Xiaolong Song et al. This is an open access article distributed under the Creative Commons Attribution License, which permits unrestricted use, distribution, and reproduction in any medium, provided the original work is properly cited.

Background. Whether additional benefit can be achieved with the use of L-carnitine (L-C) in patients with chronic heart failure (CHF) remains controversial. We therefore performed a meta-analysis of randomized controlled trials (RCTs) to evaluate the effects of L-C treatment in CHF patients. Methods. Pubmed, Ovid Embase, Web of Science, and Cochrane Library databases, Chinese National Knowledge Infrastructure (CNKI) database, Wanfang database, Chinese Biomedical (CBM) database, and Chinese Science and Technology Periodicals database (VIP) until September 30, 2016, were identified. Studies that met the inclusion criteria were systematically evaluated by two reviewers independently. Results. 17 RCTs with 1625 CHF patients were included in this analysis. $\mathrm{L}-\mathrm{C}$ treatment in CHF was associated with considerable improvement in overall efficacy $(\mathrm{OR}=3.47, P<0.01)$, left ventricular ejection fraction (LVEF) (WMD: 4.14\%, $P=0.01$ ), strike volume (SV) (WMD: $8.21 \mathrm{ml}, P=0.01$ ), cardiac output (CO) (WMD: $0.88 \mathrm{~L} / \mathrm{min}, P<0.01$ ), and E/A (WMD: $0.23, P<0.01$ ). Moreover, treatment with $\mathrm{L}-\mathrm{C}$ also resulted in significant decrease in serum levels of BNP (WMD: $-124.60 \mathrm{pg} / \mathrm{ml}, P=0.01$ ), serum levels of NT-proBNP (WMD: $-510.36 \mathrm{pg} / \mathrm{ml}, P<0.01$ ), LVESD (WMD: $-4.06 \mathrm{~mm}, P<0.01$ ), LVEDD (WMD: $-4.79 \mathrm{~mm}, P<0.01$ ), and LVESV (WMD: $-20.16 \mathrm{ml}, 95 \%$ CI: -35.65 to $-4.67, P<0.01$ ). However, there were no significant differences in all-cause mortality, 6-minute walk, and adverse events between L-C and control groups. Conclusions. L-C treatment is effective for CHF patients in improving clinical symptoms and cardiac functions, decreasing serum levels of BNP and NT-proBNP. And it has a good tolerance.

\section{Introduction}

Chronic heart failure (CHF) is a complex clinical syndrome characterized by decreased myocardial contractility, hemodynamic abnormality, and neuroendocrine activation. It is a global public health problem affecting estimated 26 million worldwide [1]. Currently, the neurohormonal antagonists (ACE-inhibitors, beta-blockers, angiotensin receptor blockers, and mineralocorticoid receptor antagonists) are recommended for CHF as cornerstones [2, 3]. However, it remains a leading cause of morbidity and mortality throughout the world.

Recently, there has been a growing appreciation of the complex metabolic processes underlying HF pathophysiology and symptoms [4]. In fact, the failing heart may be defined as "an engine out of fuel" [5]. L-carnitine is a vitaminlike and modified amino acid that plays an important role in supporting the body's metabolic activities. There is growing evidence that high concentrations of L-C provide beneficial effects in various diseases such as coronary artery disease, congestive heart failure, peripheral vascular diseases, type 2 diabetes, dyslipidemia, and hypertension [6].

However, the clinical guidelines about nutritional supplements in different countries are not consistent. In Chinese guideline, nutritional supplements (trimetazidine, coenzyme Q10, and L-C) may be helpful to CHF [7]. But, as a treatment of heart failure, L-C has not been recommended in patients with current or prior symptoms of heart failure with reduced ejection fraction and heart failure with preserved ejection fraction in the American guideline [3]. The recommendation 
of nutritional supplements has not been proposed in the European guideline [2].

In addition, two meta-analyses of RCTs has been performed to assess the therapeutic effects of $\mathrm{L}-\mathrm{C}$ in the secondary prevention of cardiovascular disease $[8,9]$, but there is no meta-analysis of RCTs in CHF. Over the past few decades, several small RCTs have been conducted to evaluate the effects of L-C treatment in patients with CHF. Thus, we performed a meta-analysis of RCTs with critical inclusion and exclusion criteria to evaluate the efficacy and tolerance of LC.

\section{Materials and Methods}

2.1. Search Strategy. We searched Pubmed, Ovid Embase, Web of Science, and Cochrane Library databases, Chinese National Knowledge Infrastructure (CNKI) database, Wanfang database, Chinese Biomedical (CBM) database, Chinese Science and Technology Periodicals database (VIP) until September 30, 2016. The following medical subject headings were used: "L-carnitine," "carnitine," "levocarnitine," "novain," "L-cthernitine," "Vitamin BT," "Bicarnesine," "heart failure," "cardiac dysfunction," "cardiac insufficiency," "cardiac inadequacy," "cardiomyopathy," and "ventricular dysfunction." Electronic searches were supplemented with manual searches of reference lists of all retrieved review articles, primary studies, and abstracts from meetings to identify other studies not found in the electronic searches. Literature was searched by two authors (X. Song and Z. Yang) independently. The search was limited to human subjects, with no restriction for language.

2.2. Study Selection. Two authors independently selected trials and discussed with each other when inconsistencies were found. Articles that meet the following criteria were included: (1) study types, randomized controlled trials; (2) participants, chronic heart failure patients (age $\geq 18$ years); (3) interventions, L-C with placebo, routine, or conventional treatment; (4) outcome measures, studies that used one or more of the following measurements were eligible: all-cause mortality, cardiovascular events, New York Heart Association (NYHA) classification, overall efficacy, exercise capacity (i.e., 6-minute walk), changes in cardiac function parameters (i.e., left ventricular ejection fraction (LVEF), strike volume (SV), cardiac output (CO), E/A, left ventricular end-systolic dimension (LVESD), left ventricular end-diastolic dimension (LVEDD), and left ventricular end-systolic volume (LVESV)), brain natriuretic peptide (BNP), N-terminal probrain natriuretic peptide (NT-proBNP), and adverse events. (5) Full texts available. Studies without randomized method from CNKI, CBM, and VIP were excluded. Studies that included other nutritional supplements (i.e., trimetazidine, coenzyme Q10) were excluded. Nonrandomized evaluations, pharmacokinetic studies, animal/laboratory studies, and general reviews were excluded, and duplicated publications reporting the same groups of patients were also excluded (Figure S1 in Supplementary Material available online at https://doi.org/10.1155/2017/6274854).
2.3. Quality Assessment. The methodological qualities of the included RCTs were assessed according to Cochrane Collaboration's tool described in Handbook version 5.1.0 [10]. Two authors (X. Song and Z. Yang) assessed the quality independently, and inconsistency was discussed with a third review author (H. Zhou) who acted as an arbiter.

2.4. Data Extraction. Two researchers read the full texts independently and extracted the following contents: publication data (first author's name, year of publication), study characteristic (study design, sample size, follow-up duration, inclusion criteria, and endpoints), patient characteristics (age, gender, NYHA classification, cardiac histology, and LVEF), treatment protocol (L-C dose), and outcome measures (all-cause mortality, overall efficacy, NYHA classification, 6-minute walk, LVEF, SV, CO, E/A, LVEDD, LVESD, LVESV, BNP, NT-proBNP, and adverse events). Authors were contacted by e-mail for additional information if data were unavailable.

2.5. Statistical Methods. Data were processed in accordance with the Cochrane Handbook [10]. Intervention effects were expressed with odds ratios (ORs) and associated 95\% confidence intervals (CIs) for dichotomous data. By contrast, the effects were expressed with mean differences and 95\% CIs for continuous data. Statistical heterogeneity was measured using $I^{2}$ statistic and $I^{2}$ statistic with significance set at $I^{2}$ greater than $50 \%$ [11].

The fixed-effects model was first used for meta-analyses. The random-effects model was used in the presence of heterogeneity. Description analysis was performed when the quantitative data could not be pooled. Intention-totreat (ITT) principle was used. Review Manage (v.5.1; the Cochrane Collaboration) was used for data analysis.

\section{Results}

3.1. Study and Patient Characteristics. The flow of selecting studies for the meta-analysis is shown in Figure S1. Briefly, among the initial 2870 reports, 468 articles were retrieved for detailed evaluation, and 17 RCTs [12-28] enrolling 1625 participants that fulfilled the inclusion criteria were finally analyzed. The study and patient characteristics are shown in Table 1. L-C dosage ranged from 1.5 to $6 \mathrm{~g} /$ day and follow-up periods from 7 days to 3 years.

3.2. Methodological Quality Assessment. All studies included in this meta-analysis were randomized controlled trials. The quality assessment of the included RCTs is shown in Figure 1. Four studies $[12,13,21,26]$ did not report the method of randomization, whereas the others reported a randomization number sequence or adaptive minimization randomization scheme. There were three studies $[13,17,26]$ using the blind method; blind methods of all the other studies are unclear. One study [21] had high performance bias for the reason that more than $20 \%$ of patients were lost to follow-up. Selective reporting was found in three studies $[13,21,26]$ because they did not present the ITT analysis data. These studies had 


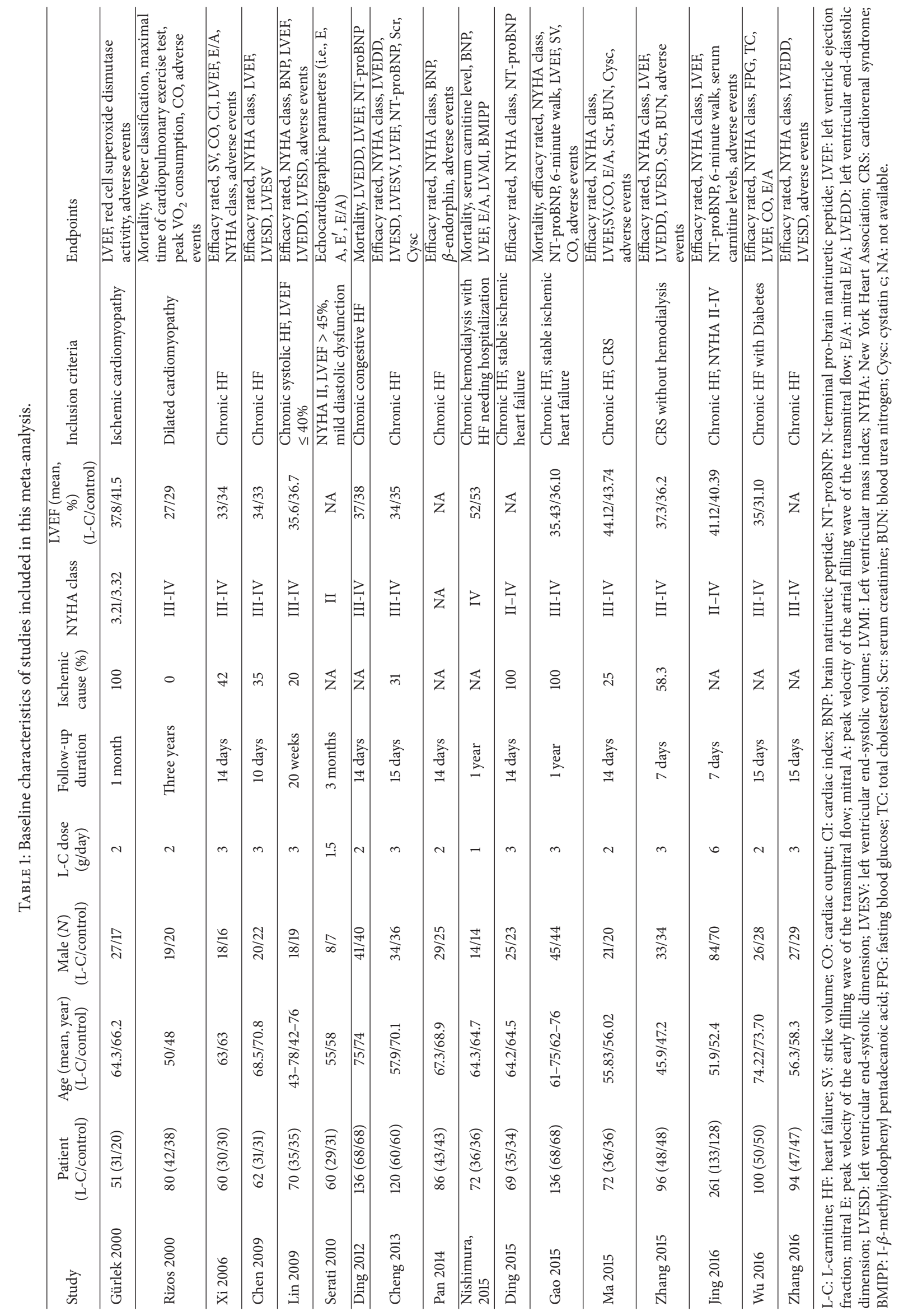




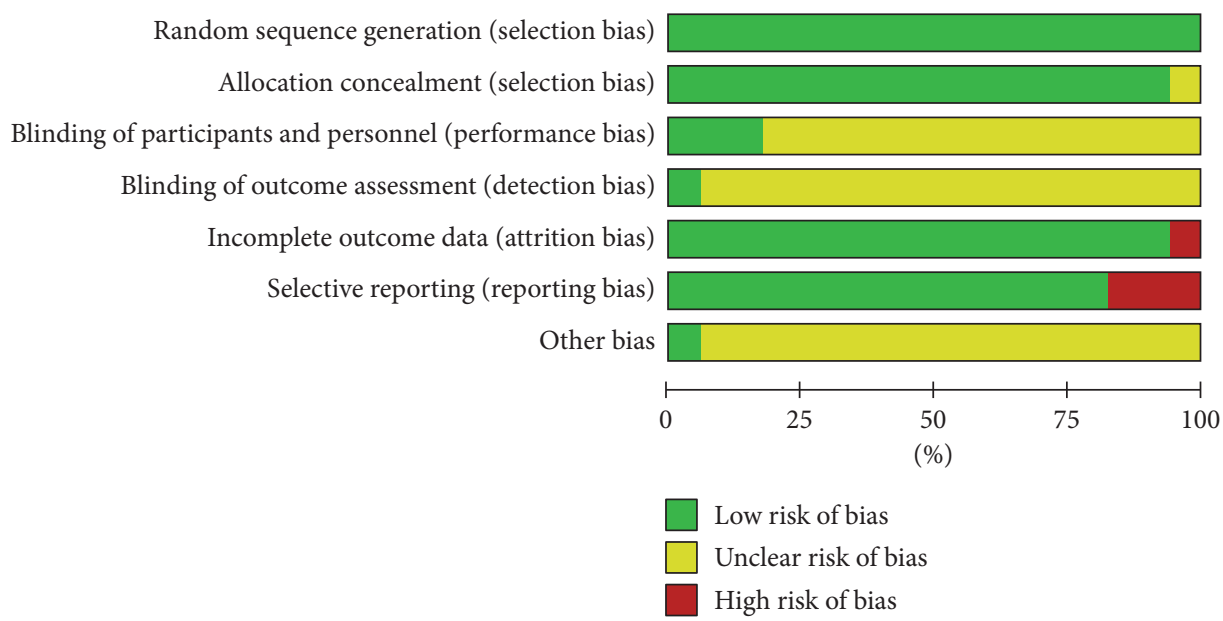

(a) Risk of bias graph: review authors' judgements about each risk of bias item presented as percentages across all included studies

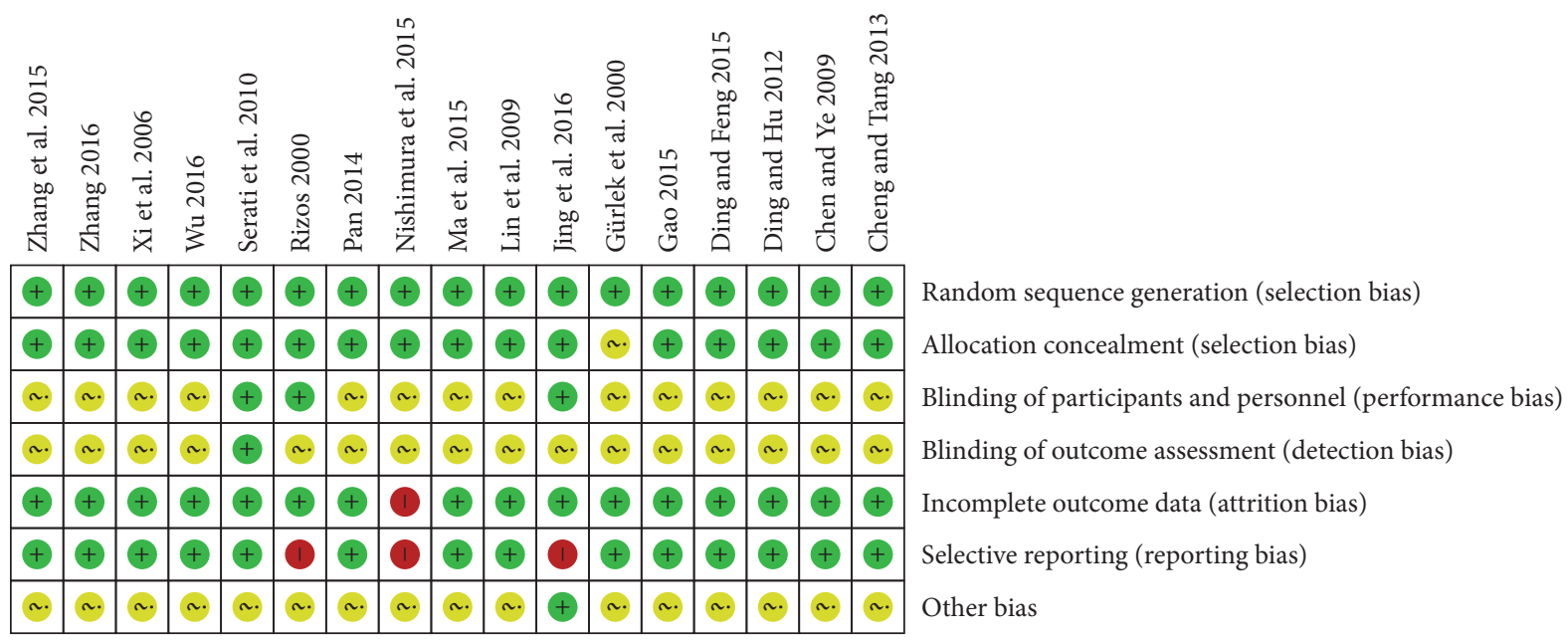

(b) Risk of bias summary: review authors' judgements about each risk of bias item for each included study

FiguRE 1: Risk of bias assessment.

high performance bias and detection bias. The other potential biases were unclear in these trials (Figure 2). Because most of the studies were conducted in China, we cautiously drew the conclusion that publication bias might have been present in the meta-analysis. The differences of treatment period would influence the outcomes of chronic heart failure patients. Eight studies [14, 18-20, 22, 24, 27, 28] had a treatment period of 2 weeks, and the follow-up period of other studies still ranged variously. In this condition, we conducted a subgroup analysis in these studies with 2 -week treatment period (Figure S3).

3.3. All-Cause Mortality for Cardiac Causes. Four studies [13, $18,21,23]$ reported all-cause mortality. As shown in Figure 2, no significant differences were found in heterogeneity in both per-protocol (PP) and ITT analysis $\left(I^{2}=6 \%, P=0.36\right.$ and $I^{2}=7 \%, P=0.36$, resp.). The results of $\mathrm{PP}$ analysis showed that all-cause mortality in the L-C group was not lower than control $(\mathrm{OR}=0.48,95 \% \mathrm{CI}$ : 0.21 to $1.06, P=$ 0.07 , Figure $2(\mathrm{a})$ ). ITT analysis also showed no differences in all-cause mortality between heart failure patients in both groups $(\mathrm{OR}=0.49,95 \% \mathrm{CI}: 0.22$ to $1.08, P=0.08$, Figure 2(b)).

3.4. Functional Capacity. The improvement in cardiac function (decreased NYHA class) was rated as overall efficacy. The endpoint was a decrease of at least one NYHA class, with efficacy rated as effective (decrease of two classes, decrease of one class) or ineffective (no class change). The overall efficacy consisted of excellent and effective rate. Twelve studies [14-16, 19, 20, 22-28] reported overall efficacy; there were no significant differences in heterogeneity $\left(I^{2}=0 \%\right.$, $P=0.45)$. Meta-analysis indicated that patients who received L-C treatment had higher overall efficacy than those in control group $(\mathrm{OR}=3.47,95 \% \mathrm{CI}: 2.49$ to $4.82, P<0.01$, Figure 3(a)). Subgroup analysis of 2 -week treatment period also demonstrated that overall efficacy of L-C treatment was higher than the control group $(\mathrm{OR}=5.11,95 \% \mathrm{CI}$ : 2.87 to $9.10, P<0.01$, Figure S3). Considering significant heterogeneity between studies $[23,26]$ when we compared difference values about $\Delta 6$-minute walk, meta-analysis with 


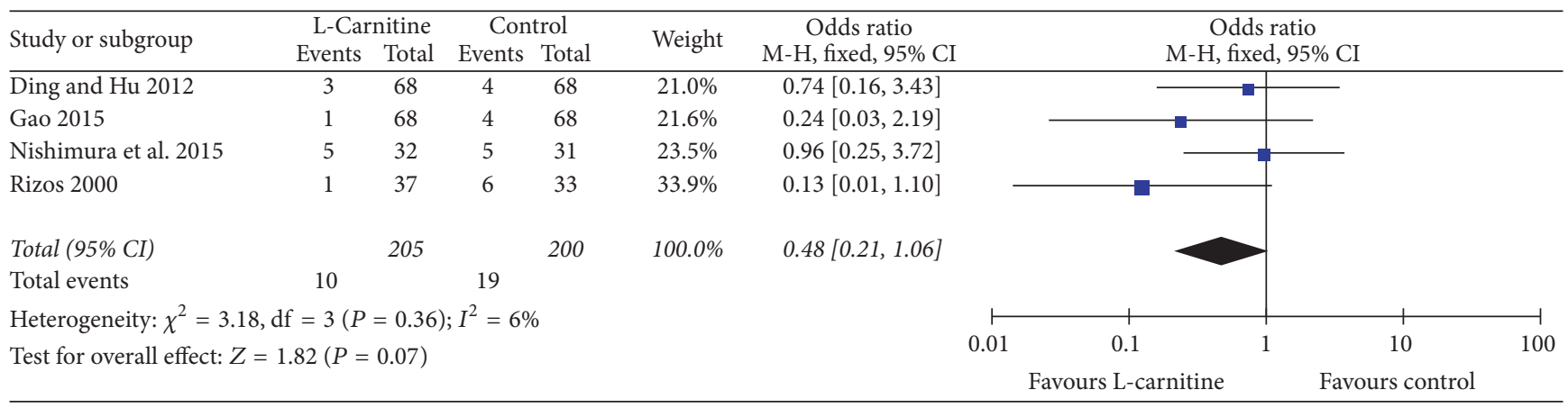

(a) PP analysis

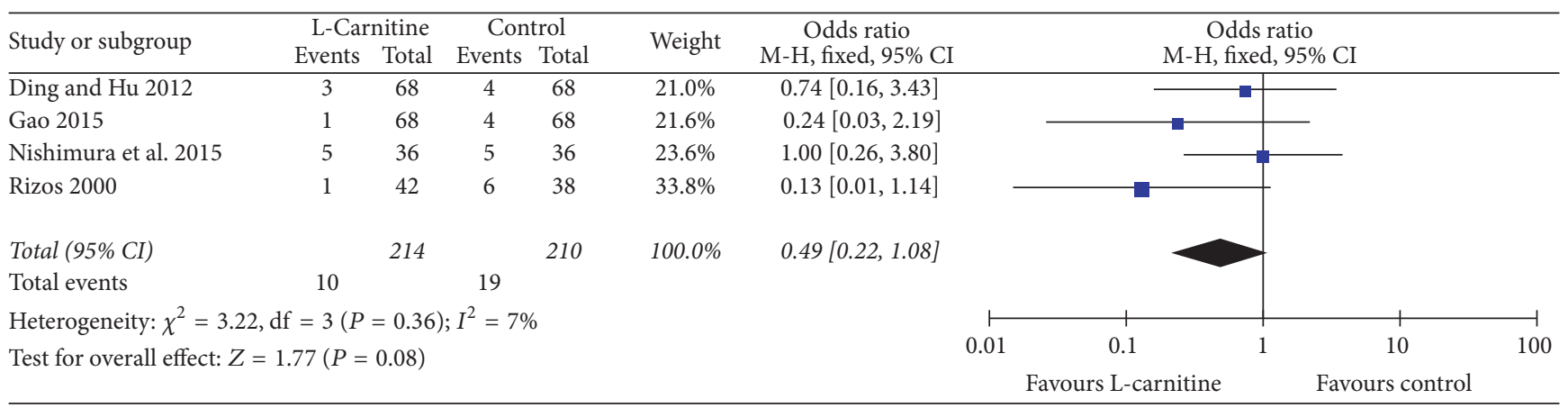

(b) ITT analysis

FIGURE 2: Forest plots for all-cause mortality.

random-effect model revealed that there were no significant differences in improvement of exercise tolerance between the two treatments (WMD: $45.41 \mathrm{~m}, 95 \% \mathrm{CI}:-14.46$ to 105.29 , $P=0.14$, Figure 3(b)).

3.5. Serum Markers. Heterogeneity was significant among the included studies $[16,18-23,26]$ in which there were changes in serum levels of BNP and NT-proBNP $\left(I^{2}=97 \%\right.$, $P<0.01$ and $I^{2}=99 \%, P<0.01$, resp.). Thus, a randomeffects model was applied; we found that in serum levels of BNP and NT-proBNP were significantly decreased in the LC group compared with those in the control group (WMD: $-124.60 \mathrm{pg} / \mathrm{ml}, 95 \% \mathrm{CI}:-220.49$ to $-28.71, P=0.01$; WMD: $-510.36 \mathrm{pg} / \mathrm{ml}, 95 \% \mathrm{CI}:-785.42$ to $-235.30, P<0.01$, resp., Figure 4). Subgroup analysis indicated that levels of NT-proBNP were significantly decreased in the L-C group (WMD: $-612.44 \mathrm{pg} / \mathrm{ml}, 95 \% \mathrm{CI}:-829.41$ to $-395.47, P<0.01$, Figure S3).

3.6. Left Ventricular Structure and Function. Twelve studies $[12,14-16,18,19,21,23-27]$ provided data on LVEF, considering that significant heterogeneity was found among the included studies $\left(I^{2}=70 \%, P<0.01\right)$; we used a random-effect model and a profound improvement in LVEF was observed in patients who received L-C therapy (WMD: 4.14\%, 95\% CI: 2.34 to 5.93, $P=0.01$, Figure 5(a)). As shown in Figures 5(b) and 5(c), no significant differences were found in heterogeneity in both SV $[14,23,24]$ and CO [14, 23, 24, 27] analysis $\left(I^{2}=0 \%, P=0.74\right.$ and $I^{2}=0 \%, P=0.65$, resp.).
SV and CO were significantly higher in patients who received L-C therapy than control group (WMD: $8.21 \mathrm{ml}, 95 \% \mathrm{CI}$ : 6.41 to $10.01, P=0.01$; WMD: $0.88 \mathrm{~L} / \mathrm{min}, 95 \% \mathrm{CI}: 0.76$ to 1.01, $P<0.01$, resp., Figures 5(b) and 5(c)). Heterogeneity was significant among the studies $[14,17,21,24,27]$ when comparing E/A $\left(I^{2}=82 \%, P<0.01\right)$. Thus, a randomeffects model was used. Our data revealed that E/A was significantly higher for patients who received L-C treatment (WMD: $0.23,95 \%$ CI: 0.11 to $0.35, P<0.01$, Figure $5(\mathrm{~d})$ ). In addition, our results indicated that $\mathrm{L}-\mathrm{C}$ was associated with a significant drop in LVESD $[15,16,19,25,28]$ and LVEDD $[16,18,19,25,28]$ for patients (WMD: $-4.06 \mathrm{~mm}, 95 \% \mathrm{CI}$ : -6.57 to $-1.55, P<0.01$; WMD: $-4.79 \mathrm{~mm}, 95 \% \mathrm{CI}:-7.08$ to $-2.49, P<0.01$, resp., Figures 6(a) and 6(b)). We also found that LVESV $[15,19]$ was significantly decreased in response to L-C therapy (WMD: $-20.16 \mathrm{ml}, 95 \% \mathrm{CI}:-35.65$ to -4.67 , $P<0.01$, Figure 6(c)). According to subgroup analysis of 2-week treatment period, statistically significant association was found between LVEF, SV, CO, E/A, LVESD, and LVEDD (WMD: $6.63 \%$, 95\% CI: 4.78 to $8.47, P<0.01$; WMD: $9.28 \mathrm{ml}$, 95\% CI: 5.90 to $12.67, P<0.01$; WMD: $0.90 \mathrm{~L} / \mathrm{min}, 95 \% \mathrm{CI}$ : 0.78 to $1.03, P<0.01$; WMD: $0.31,95 \% \mathrm{CI}: 0.16$ to 0.46 , $P<0.01$; WMD: $-6.50 \mathrm{~mm}, 95 \% \mathrm{CI}:-8.11$ to $-4.89, P<0.01$; WMD: $-3.24 \mathrm{~mm}, 95 \% \mathrm{CI}:-5.93$ to $-0.55, P<0.01$, resp., Figure S3).

3.7. Major Adverse Events. Six studies [12, 14, 16, 24, 25, 28] reported that there were no adverse events related to L-C. Four reports $[13,20,23,26]$ contained data on specific adverse 


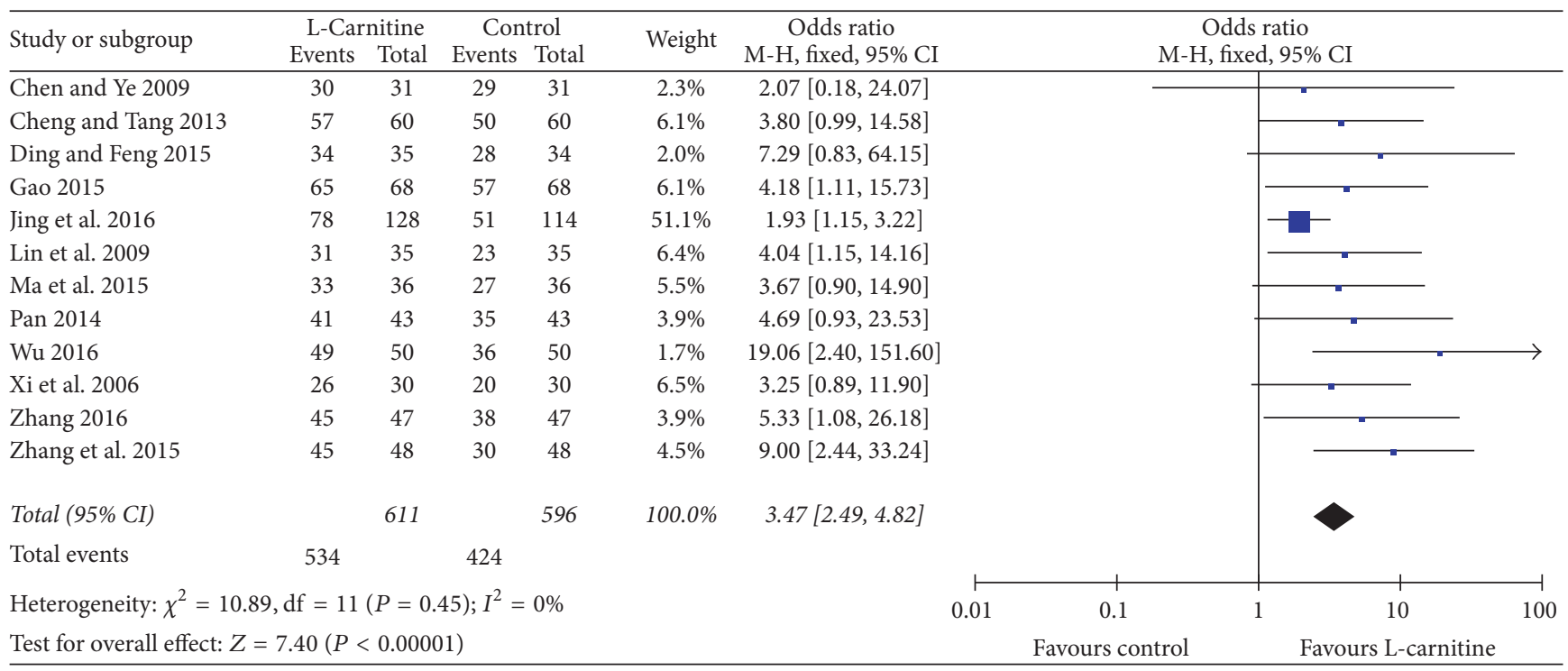

(a) Overall efficacy

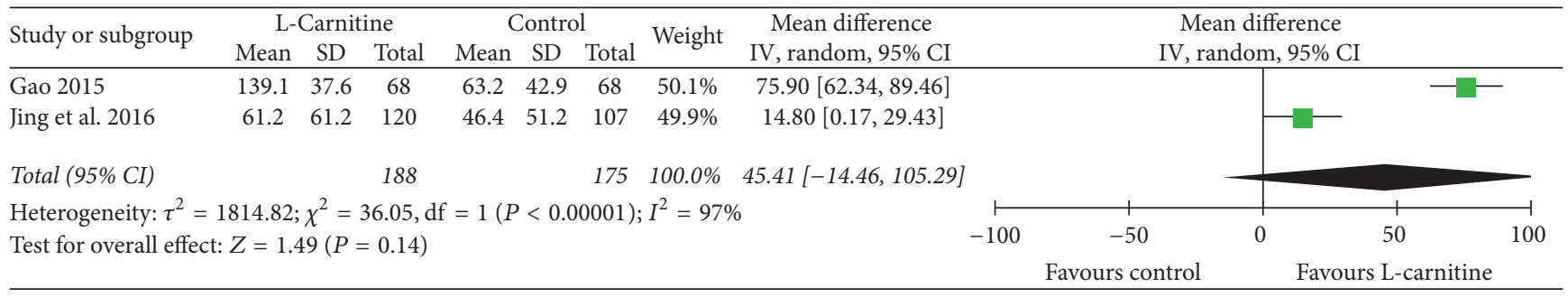

(b) $\Delta$ 6-minute walk exercise

FIGURE 3: Forest plots for functional capacity.

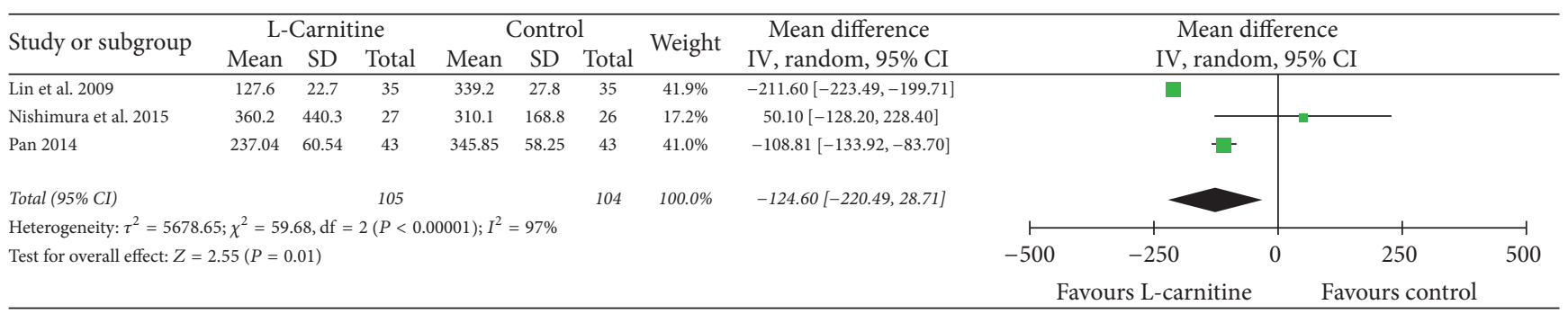

(a) BNP

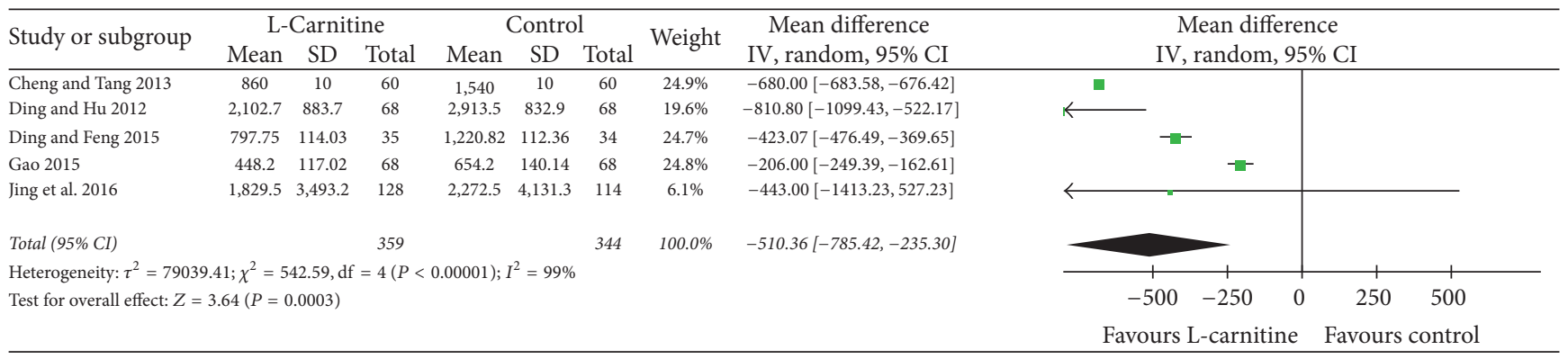

(b) NT-proBNP

FIGURE 4: Forest plots for serum markers. 


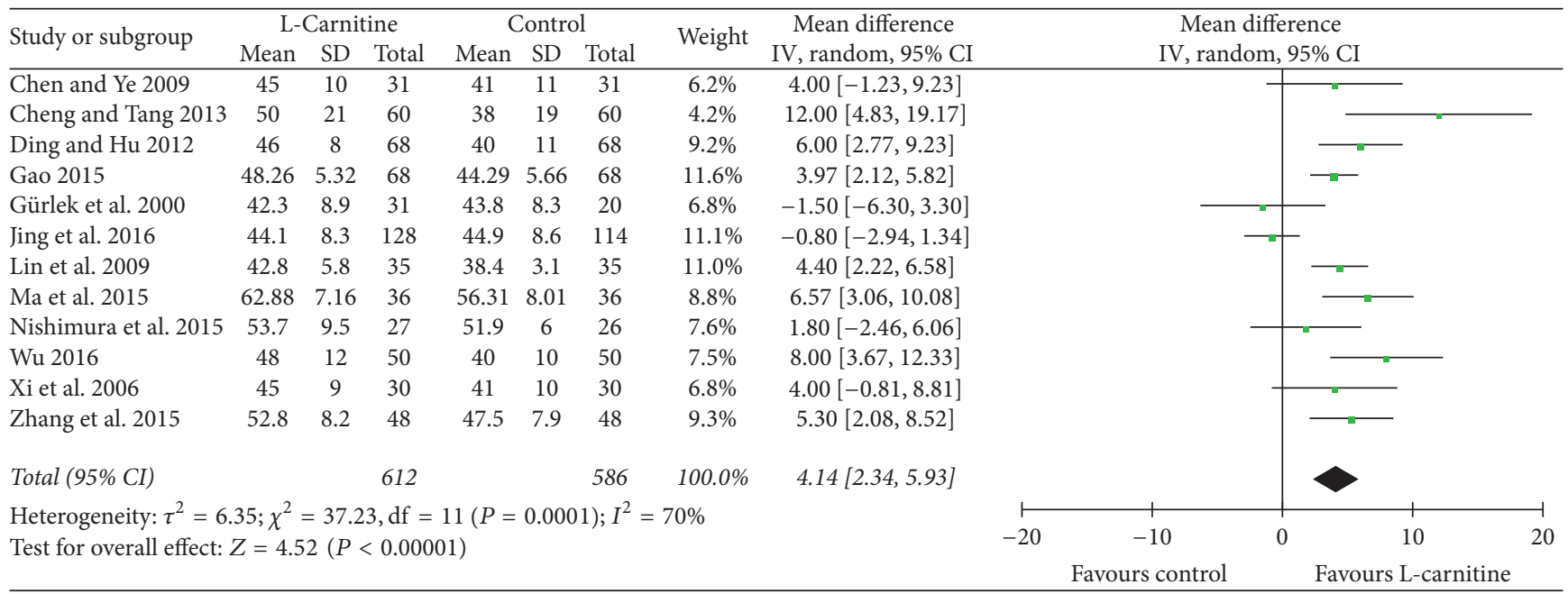

(a) LVEF

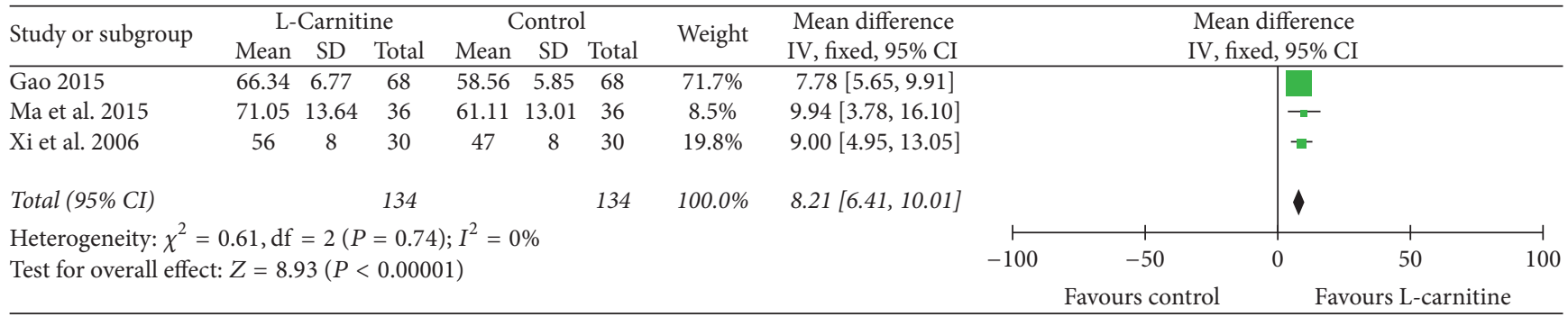

(b) SV

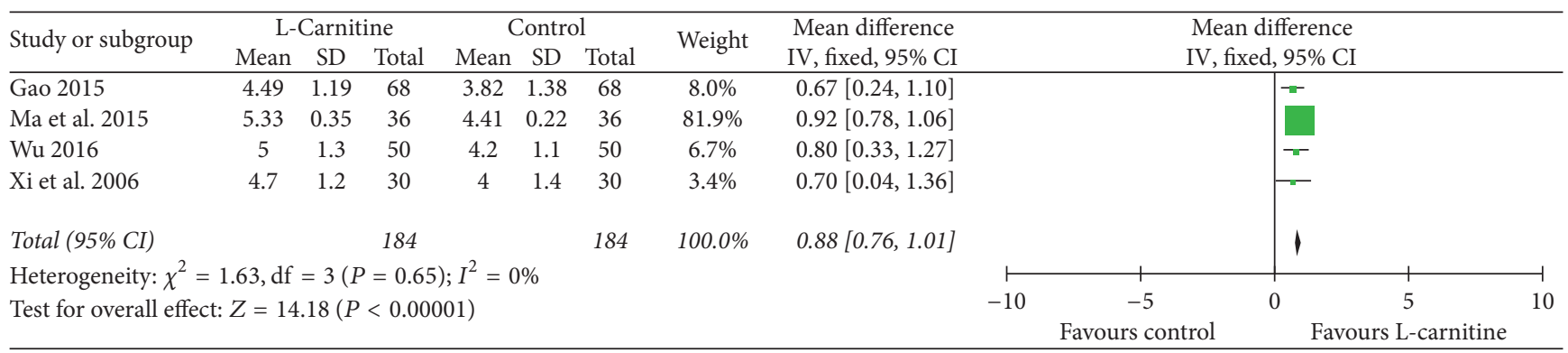

(c) $\mathrm{CO}$

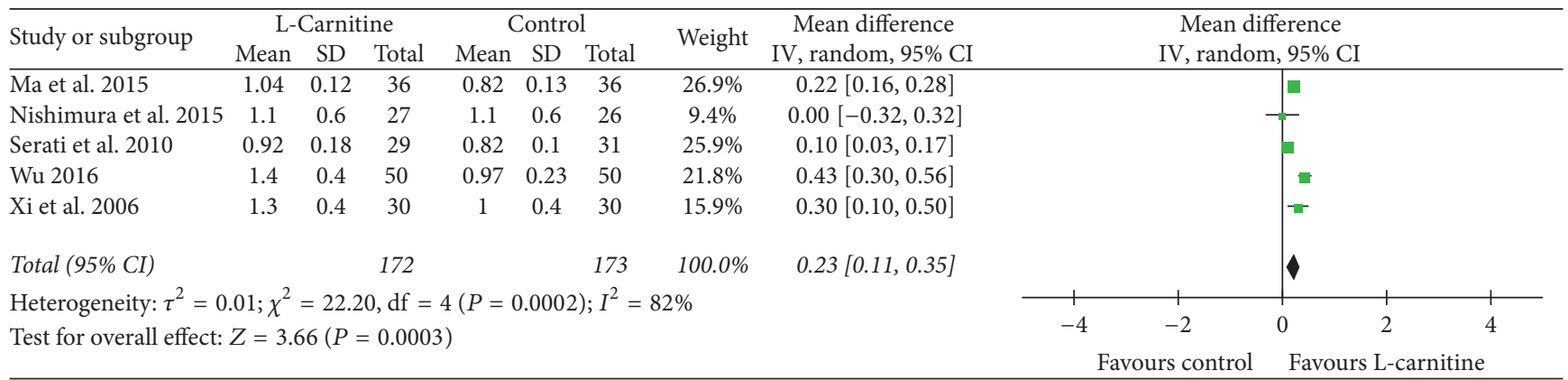

(d) $\mathrm{E} / \mathrm{A}$

FIGURE 5: Forest plots for left ventricular structure and function (I). 


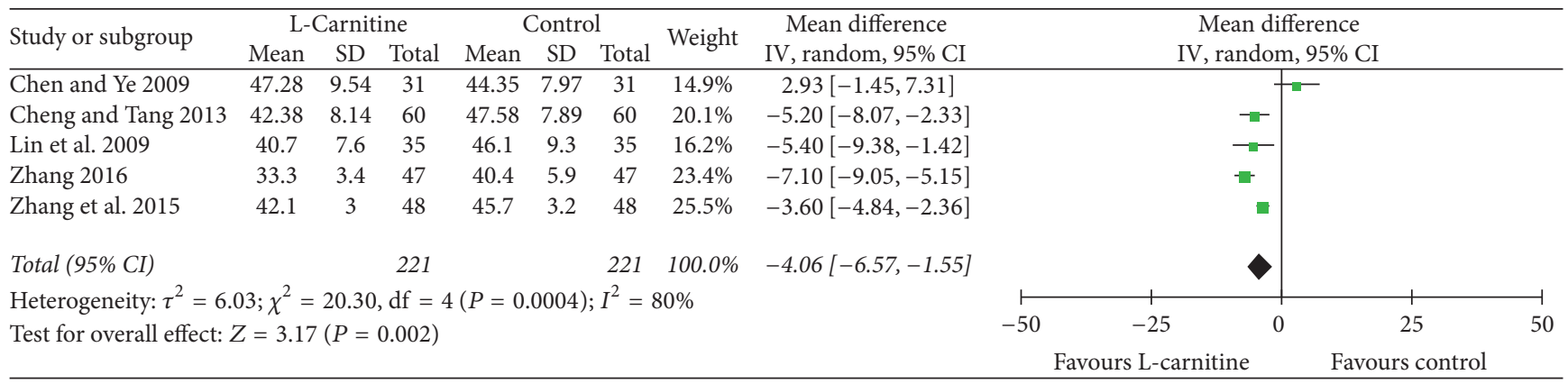

(a) LVESD

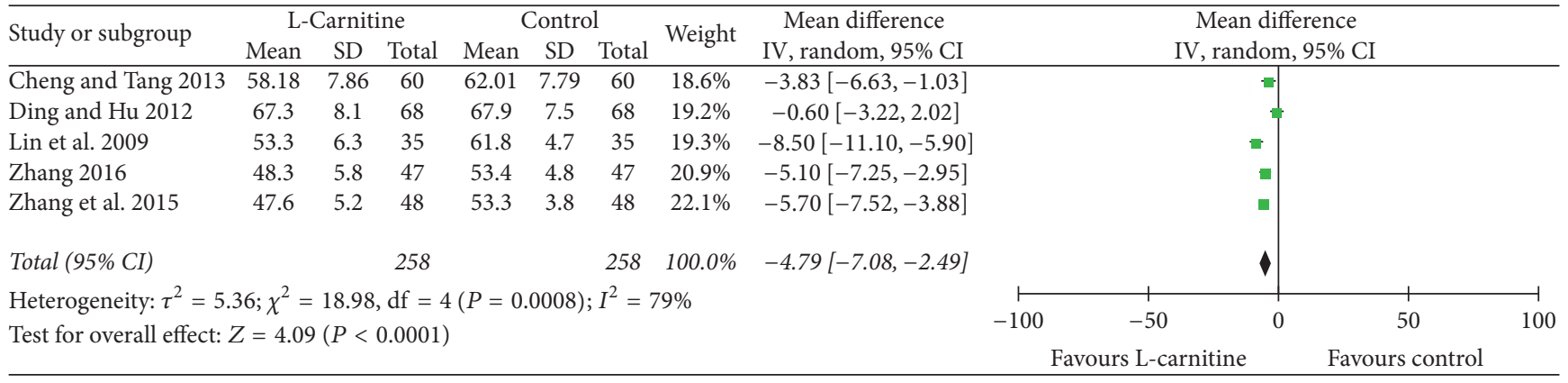

(b) LVEDD

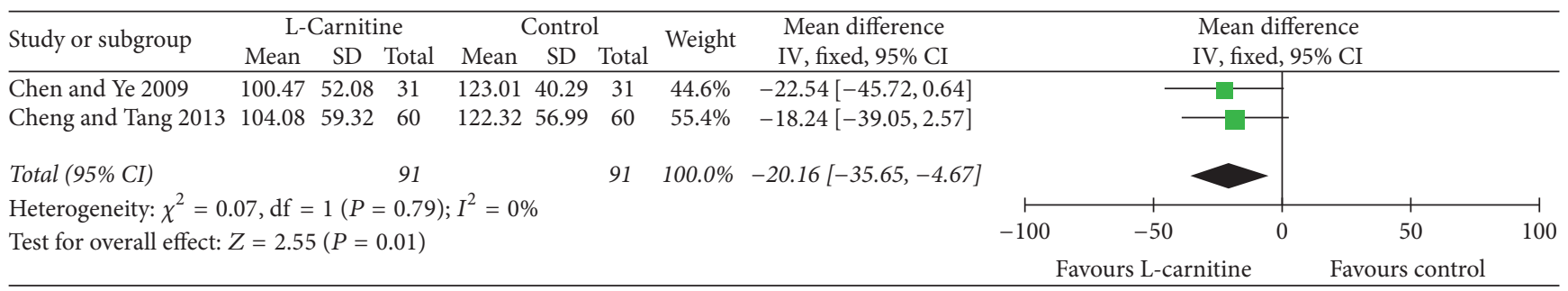

(c) LVESV

FIGURE 6: Forest plots for left ventricular structure and function (II).

events. Dry mouth and gastrointestinal problems were the major adverse events, and rash was reported by a study of Pan [20] in both L-C group (4/43) and control group (2/43). No participants have withdrawn from the study for the reason of adverse events. Meta-analysis demonstrated no differences in adverse events between patients in both groups (5.4\% versus $5.8 \%, \mathrm{OR}=0.92,95 \% \mathrm{CI}: 0.44$ to $1.92, P=0.83$, Figure S2). The other seven trials did not report adverse events.

\section{Discussion}

L. H. Opie indicated that "the heart is more than a pump. It is also an organ that needs energy from metabolism. A metabolic disease, ischaemia, should ideally be treated by metabolic therapy" [29]. CHF is currently conceived as a systemic and multiorgan syndrome with metabolic failure as basic mechanism. In fact, the failing heart may be defined as "an engine out of fuel" [5]. L-C is a natural constituent of human cells and participates in fatty acid metabolism. L-C plays an important role in lipid metabolism by acting as an obligatory cofactor for oxidation of fatty acids and facilitating the transport of long-chain fatty acids (LCFAs) across the mitochondrial membrane to provide enough adenosine triphosphate (ATP) for myocardial cells [30].

Although heart failure is not listed as one of indications in package insert of L-C, clinical application of L-C has shown significant relief of heart failure, which was confirmed in our research.

Recently, DiNicolantonio et al. (2013) [9] conducted a meta-analysis of L-C in the secondary prevention of cardiovascular disease. Compared with placebo, L-C was associated with a $27 \%$ reduction in all-cause mortality, a $65 \%$ reduction in ventricular arrhythmias, and a $40 \%$ reduction in angina in patients experiencing an acute myocardial infarction (AMI). However, our study suggests that the additional use of L-C failed to reduce all-cause mortality in CHF patients.

Chronic oral L-C supplementation has been shown to ameliorate factors associated with metabolic syndrome and cardiovascular disease, such as arterial hypertension, cholesterol levels, impaired glucose tolerance, and insulin resistance. L-C appears to be particularly suitable as a treatment for metabolic syndrome patients, who are often obese, 
insulin resistant, and hypertensive [31-33]. One included study [27] suggested that L-C can significantly ameliorate total cholesterol, blood sugar, and cardiac function and improve clinical symptoms of patients for heart failure patients with diabetes mellitus.

Our findings of this meta-analysis are that the beneficial effects have been shown by the increase of overall efficacy, LVEF, strike volume, cardiac output, and E/A, by the decrease of left ventricular end-diastolic diameter, left ventricular endsystolic diameter, and left ventricular end-systolic volume and serum levels of BNP and NT-proBNP, and with satisfactory safety. Some reports $[21,24,25]$ indicated that clinical symptom, cardiac function, and renal function in $\mathrm{CHF}$ patients with renal insufficiency were more likely to be ameliorated with L-C treatment.

According to the "energy starvation" hypothesis, which induces that insufficient ATP supply underlies the contractile dysfunction presenting in heart failure [34], it seems reasonable that $\mathrm{L}-\mathrm{C}$ improves energy metabolism in cardiomyocytes, which may finally translate into mechanical efficiency and contribute to the improvement of clinical symptoms and cardiac function. Furthermore, noteworthy is that L-C exerts cardioprotective effects through alternative mechanisms, such as oxidative stress [35], nitric oxide [36], arterial hypertension, cardiac inflammation and fibrosis [3739], and interstitial remodeling [40], as well as by improving endothelial function [41].

It is noteworthy that, based on existing conventional treatment, L-C was used in treatment of energy metabolism disorder. It acted as a supplementation to preexisting treatment rather than a replacement. Chronic heart failure is a disease that requires multitargeted and phase dependent therapeutic methods. Our research showed that L-C represents a safe and effective adjuvant therapy which, by increasing high energy phosphate for systolic and diastolic function, may have a synergistic effect with other drugs.

According to study, relatively high degree of heterogeneity was found regarding the following four indices: LVEF, BNP, NT-proBNP, and 6-minute walking distance. Through analyzing the included references, we speculated the following possible causes for the discrepancies: (1) different uses, doses of L-C utilized in these studies. (2) Different states of illness and stages of therapies of patients included in these studies. (3) Manufacturers and specifications of L-C being not clearly indicated in most of these studies.

Some limitations of our meta-analysis need to be acknowledged. Firstly, the methodological quality of included studies was less than optimal, so we were not able to exclude the potential risk of bias in these trials. Secondly, it is worth noticing that only 1625 patients were involved in the 17 RCTs, which justifies the performance of more large-scale RCTs for evaluating the impact of L-C treatment on CHF patients. Thirdly, the follow-up duration in these studies varied widely, from 7 days to 3 years. Owing to these limitations, our results are insufficient to recommend the method as a first-line treatment or to establish the quality of life and long-term results. Therefore, further research is required to more accurately assess the results of L-C for treating CHF.
In conclusion, our meta-analysis demonstrates that $\mathrm{L}-\mathrm{C}$ treatment in $\mathrm{CHF}$ patients may improve clinical symptoms and cardiac function and decrease serum levels of BNP and NT-proBNP and has good tolerance.

$\begin{array}{ll}\text { Abbreviations } \\ \text { L-C: } & \text { L-Carnitine } \\ \text { CHF: } & \text { Chronic heart failure } \\ \text { NYHA: } & \text { New York Heart Association } \\ \text { BNP: } & \text { Brain natriuretic peptide } \\ \text { NT-proBNP: } & \text { N-terminal pro-brain natriuretic peptide } \\ \text { LVEF: } & \text { Left ventricle ejection fraction } \\ \text { LVEDD: } & \text { Left ventricular end-diastolic dimension } \\ \text { LVESD: } & \text { Left ventricular end-systolic dimension } \\ \text { LVESV: } & \text { Left ventricular end-systolic volume } \\ \text { mitral E: } & \text { Peak velocity of the early filling wave of } \\ & \text { the transmitral flow } \\ \text { mitral A: } & \text { Peak velocity of the atrial filling wave of } \\ & \text { the transmitral flow } \\ \text { E/A: } & \text { Mitral E/A } \\ \text { CI: } & \text { Confidence interval } \\ \text { WMD: } & \text { Weighted mean differences. }\end{array}$

\section{Disclosure}

The funders had no role in study design, data collection and analysis, decision to publish, or preparation of the manuscript.

\section{Conflicts of Interest}

The authors declare that they have no competing interests.

\section{Acknowledgments}

This work was partly supported by the National Natural Science Foundation of China (Research Grants no. 81673753 and no. 81373625 to H. Zhou).

\section{References}

[1] A. P. Ambrosy, G. C. Fonarow, J. Butler et al., "The global health and economic burden of hospitalizations for heart failure: lessons learned from hospitalized heart failure registries," Journal of the American College of Cardiology, vol. 63, no. 12, pp. 1123-1133, 2014.

[2] J. Špinar, J. Hradec, L. Špinarová, and J. Vítovec, "Summary of the 2016 ESC Guidelines on the diagnosis and treatment of acute and chronic heart failure. Prepared by the Czech Society of Cardiology," Cor et Vasa, vol. 58, no. 5, pp. e530-e568, 2016.

[3] C. W. Yancy, M. Jessup, B. Bozkurt et al., "2013 ACCF/AHA guideline for the management of heart failure: executive summary: a report of the American college of cardiology foundation/American Heart Association task force on practice guidelines," Circulation, vol. 128, no. 16, pp. 1810-1852, 2013.

[4] W. Doehner, M. Frenneaux, and S. D. Anker, "Metabolic impairment in heart failure: the myocardial and systemic perspective," 
Journal of the American College of Cardiology, vol. 64, no. 13, pp. 1388-1400, 2014.

[5] S. Neubauer, "The failing heart-an engine out of fuel," New England Journal of Medicine, vol. 356, no. 11, pp. 1140-1151, 2007.

[6] R. Ferrari, E. Merli, G. Cicchitelli, D. Mele, A. Fucili, and C. Ceconi, "Therapeutic effects of L-carnitine and propionyl-Lcarnitine on cardiovascular diseases: a review," Annals of the New York Academy of Sciences, vol. 1033, pp. 79-91, 2004.

[7] Chinese Society of Cardiology of Chinese Medical Association, "Editorial Board of Chinese Journal of Cardiology. Guidelines for the diagnosis and management of chronic heart failure 2014," Chinese Journal of Cardiology, vol. 42, no. 2, pp. 98-122, 2014 (Chinese).

[8] R. Shang, Z. Sun, and H. Li, "Effective dosing of 1-carnitine in the secondary prevention of cardiovascular disease: a systematic review and meta-analysis," BMC Cardiovascular Disorders, vol. 14, pp. 88-96, 2014.

[9] J. J. DiNicolantonio, C. J. Lavie, H. Fares, A. R. Menezes, and J. H. O'Keefe, "L-carnitine in the secondary prevention of cardiovascular disease: systematic review and meta-analysis," Mayo Clinic Proceedings, vol. 88, no. 6, pp. 544-551, 2013.

[10] J. P. T. Higgins and S. Green, Eds., Cochrane Handbook for Systematic Reviews of Interventions Version 5.1.0, The Cochrane Collaboration, 2011, http://handbook.cochrane.org/.

[11] J. P. T. Higgins and S. G. Thompson, "Quantifying heterogeneity in a meta-analysis," Statistics in Medicine, vol. 21, no. 11, pp. 15391558, 2002.

[12] A. Gürlek, E. Tutar, E. Akçil et al., "The effects of L-carnitine treatment on left ventricular function and erythrocyte superoxide dismutase activity in patients with ischemic cardiomyopathy," European Journal of Heart Failure, vol. 2, no. 2, pp. 189-193, 2000.

[13] I. Rizos, "Three-year survival of patients with heart failure caused by dilated cardiomyopathy and L-carnitine administration," American Heart Journal, vol. 139, no. 2, pp. S120-S123, 2000.

[14] H. Xi, W. Liang, J. Wu, and Z. Tan, "Clinical research of L-carnitine treatment on chronic congestive heart failure," Practical Journal of Cardiac Cerebral Pneumal and Vascular Disease, vol. 14, no. 9, pp. 714-715, 2006 (Chinese).

[15] L. Chen and W. Ye, "Clinical research of L-carnitine treatment on sixty-two patients with chronic congestive heart failure," China Foreign Medical Treatment, vol. 22, article 47, 2009 (Chinese).

[16] C. Lin, J. Li, J. Tang, and P. Yang, "Effects of levocarnitine among patients with chronic heart failure," Zhejiang Practical Medicine, vol. 14, no. 2, pp. 109-110, 2009 (Chinese).

[17] A. R. Serati, M. R. Motamedi, S. Emami, P. Varedi, and M. R. Movahed, "L-carnitine treatment in patients with mild diastolic heart failure is associated with improvement in diastolic function and symptoms," Cardiology, vol. 116, no. 3, pp. 178-182, 2010.

[18] C. Ding and X. Hu, "The effectiveness of L-carnitine treatment on senile congestive heart failure," Chinese Journal of Gerontology, vol. 32, no. 13, pp. 2881-2882, 2012 (Chinese).

[19] L. Cheng and X.-F. Tang, "Influence of levocarnitine on heart function and endocrine among patients with heart failure," Chinese Journal of Epidemiology, vol. 34, no. 6, pp. 630-632, 2013.

[20] X. Pan, "Clinical research of L-carnitine for elderly patients with chronic congestive heart failure," Zhongguo Ji Ceng Yi Yao, vol. 21, no. 23, pp. 3550-3552, 2014 (Chinese).
[21] M. Nishimura, T. Tokoro, T. Takatani et al., "Effects of intravenous L-carnitine on myocardial fatty acid imaging in hemodialysis patients: responders or non-responders to Lcarnitine," SpringerPlus, vol. 4, pp. 353-362, 2015.

[22] J. Ding and Q. Feng, "The efficiency assessment of intravenous L-carnitine on elderly patients with heart failure of coronary heart disease," Chinese Journal of Convalescent Medicine, vol. 24, no. 7, pp. 728-729, 2015 (Chinese).

[23] L. Gao, "Clinical efficiencies of levocarnitine in the treatment of ischemic cardiomyopathy heart failure and its effect on myocardial cell function," Journal of Bengbu Medical College, vol. 40, no. 12, pp. 1643-1645, 2015 (Chinese).

[24] J. Ma, X. Zhou, and X. Li, “The influence of L-carnitine on patients' cardiac function and renal function with resulting from chronic heart failure," Chinese Journal of Gerontology, vol. 35, no. 20, pp. 5761-5762, 2015 (Chinese).

[25] W. Zhang, Z. Dai, J. Zhou et al., "Clinical research of Lcarnitine treatment on patients of cardiorenal syndrome without hemodialysis," Chinese Journal of Clinical Rational Drug Use, vol. 8, no. 8C, pp. 61-62, 2015 (Chinese).

[26] Z.-C. Jing, B.-X. Wu, J.-Q. Peng et al., "Effect of intravenous 1-carnitine in Chinese patients with chronic heart failure," European Heart Journal, Supplement, vol. 18, pp. A27-A36, 2016.

[27] W. Wu, "The clinical effects of intravenous L-carnitine in elderly patients with heart failure of diabetes," Xin Xue Guan Bing Fang Zhi Zhi Shi, vol. 6, pp. 84-85, 2016 (Chinese).

[28] K. Zhang, "Clinical effects and safety of intravenous L-carnitine treatment on patients of chronic heart failure," Guide of China Medicine, vol. 14, no. 4, pp. 153-154, 2016 (Chinese).

[29] L. H. Opie, "Proof that glucose-insulin-potassium provides metabolic protection of ischaemic myocardium?" Lancet, vol. 353, no. 9155, pp. 768-769, 1999.

[30] A. P. Wong, A. Niedzwiecki, and M. Rath, "Myocardial energetics and the role of micronutrients in heart failure: a critical review," American Journal of Cardiovascular Disease, vol. 6, no. 3, pp. 81-92, 2016.

[31] Z. T. Bloomgarden, "Obesity, hypertension, and insulin resistance," Diabetes Care, vol. 25, no. 11, pp. 2088-2097, 2002.

[32] R. H. Eckel, K. Alberti, S. M. Grundy, and P. Z. Zimmet, "The metabolic syndrome," The Lancet, vol. 375, no. 9710, pp. 181-183, 2010.

[33] R. A. DeFronzo, "Pathogenesis of type 2 diabetes mellitus," Medical Clinics of North America, vol. 88, no. 4, pp. 787-835, 2004.

[34] A. M. Katz, "Is the failing heart energy depleted?" Cardiology Clinics, vol. 16, no. 4, pp. 633-644, 1998.

[35] J. L. Flanagan, P. A. Simmons, J. Vehige, M. D. Willcox, and Q. Garrett, "Role of carnitine in disease," Nutrition and Metabolism, vol. 7, article 30, 2010.

[36] A. Koc, T. Ozkan, A. Z. Karabay, A. Sunguroglu, and F. Aktan, "Effect of L-carnitine on the synthesis of nitric oxide in RAW 264.7 murine macrophage cell line," Cell Biochemistry and Function, vol. 29, no. 8, pp. 679-685, 2011.

[37] A. J. Blanca, M. V. Ruiz-Armenta, S. Zambrano et al., "Inflammatory and fibrotic processes are involved in the cardiotoxic effect of sunitinib: protective role of L-carnitine," Toxicology Letters, vol. 241, pp. 9-18, 2016.

[38] S. Zambrano, A. J. Blanca, M. V. Ruiz-Armenta et al., "Lcarnitine protects against arterial hypertension-related cardiac fibrosis through modulation of PPAR- $\gamma$ expression," Biochemical Pharmacology, vol. 85, no. 7, pp. 937-944, 2013. 
[39] Y. Omori, T. Ohtani, Y. Sakata et al., "L-Carnitine prevents the development of ventricular fibrosis and heart failure with preserved ejection fraction in hypertensive heart disease," Journal of Hypertension, vol. 30, no. 9, pp. 1834-1844, 2012.

[40] A. Orlandi, A. Francesconi, A. Ferlosio et al., "Propionyl-Lcarnitine prevents age-related myocardial remodeling in the rabbit," Journal of Cardiovascular Pharmacology, vol. 50, no. 2, pp. 168-175, 2007.

[41] S. G. Dzugkoev, I. V. Mozhaeva, M. A. Otiev, O. I. Margieva, and F. S. Dzugkoeva, "Effect of L-carnitine, afobazole and their combination with L-arginine on biochemical and histological indices of endothelial dysfunctions in cobalt intoxication in rats," Patologicheskaia fiziologiia i eksperimental'naia terapiia, vol. 59, no. 2, pp. 70-75, 2015. 


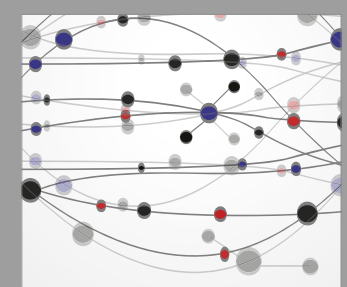

The Scientific World Journal
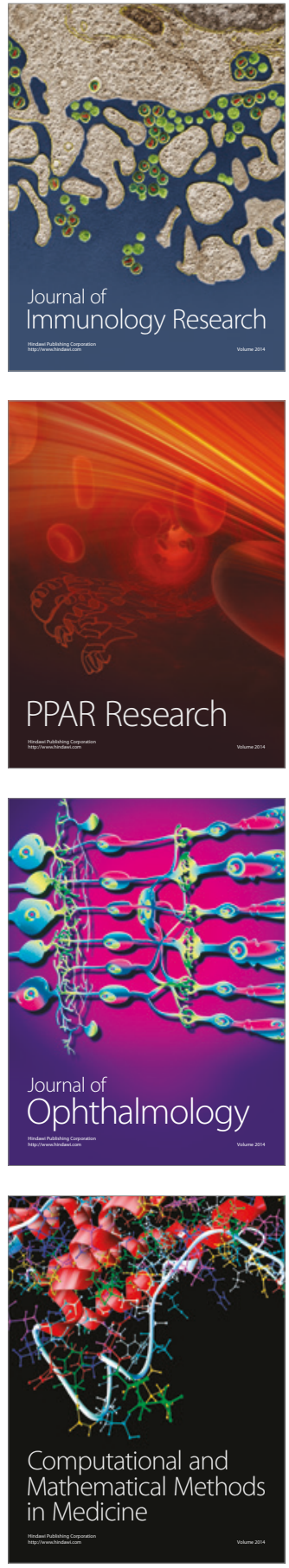

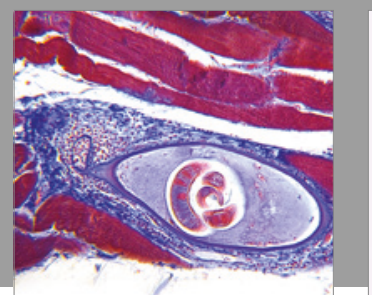

Gastroenterology Research and Practice
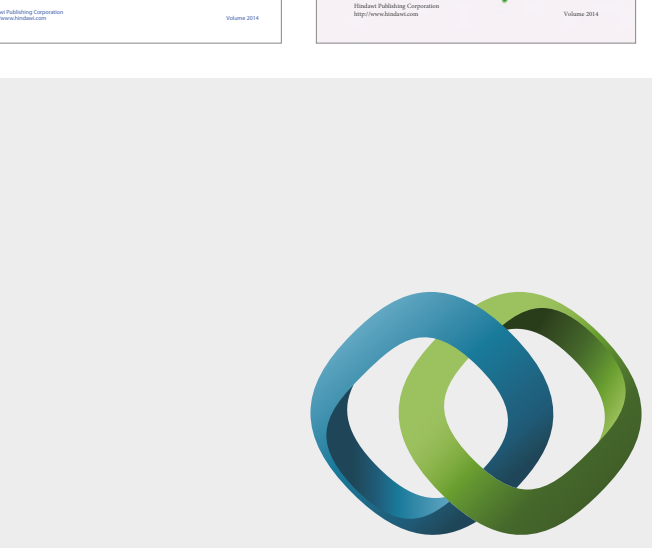

\section{Hindawi}

Submit your manuscripts at

https://www.hindawi.com
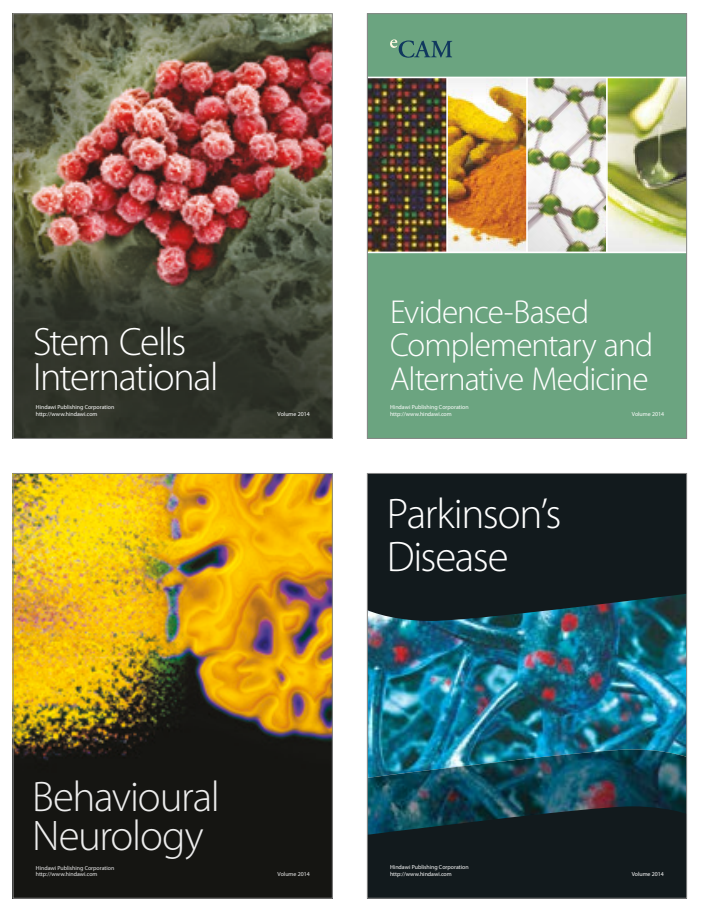
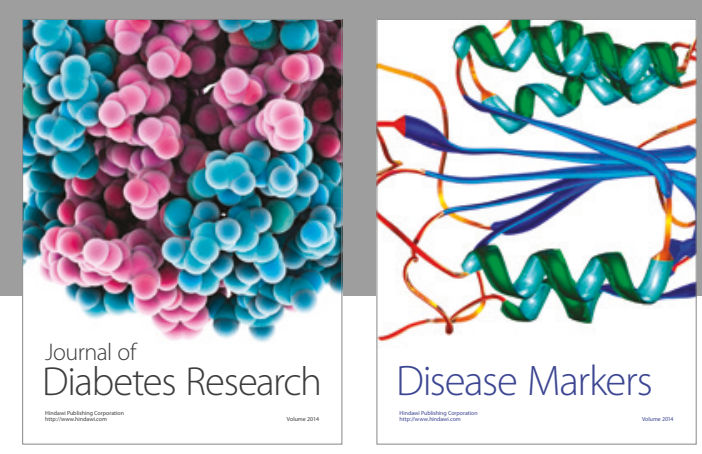

Disease Markers
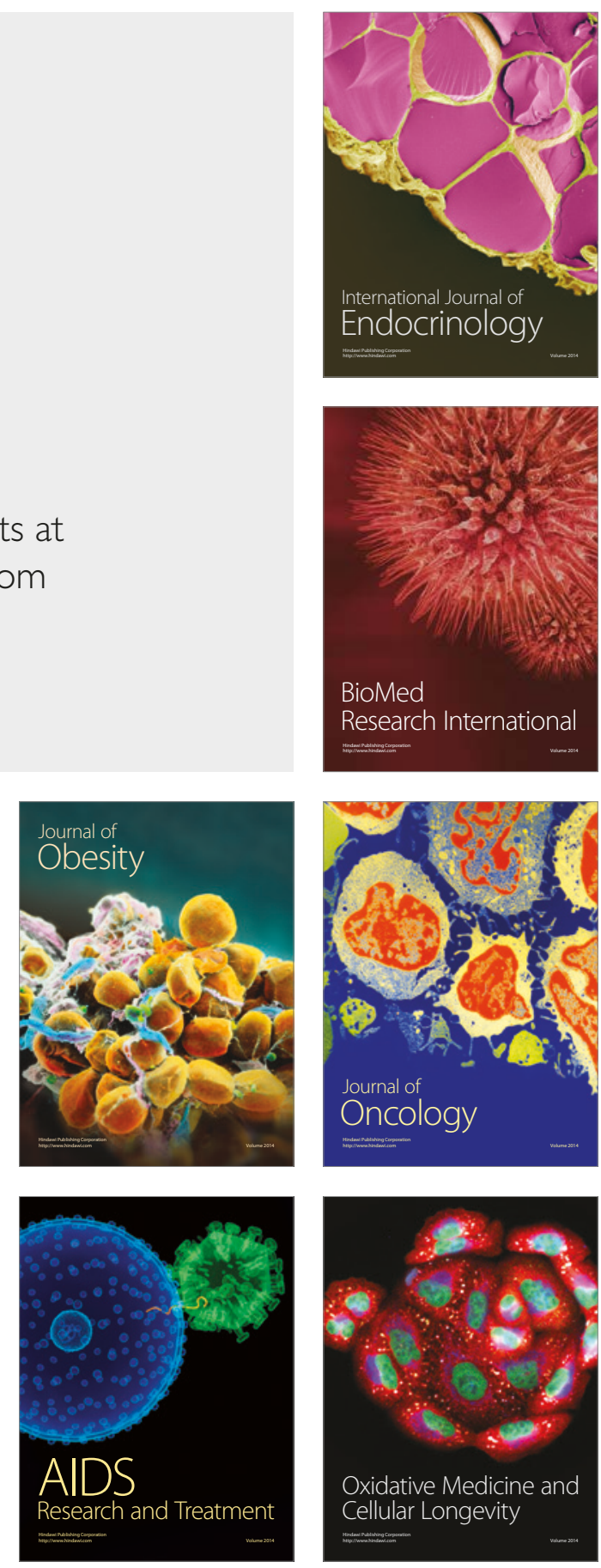